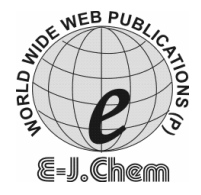

http://www.e-journals.net
ISSN: 0973-4945; CODEN ECJHAO

E-Journal of Chemistry 2010, 7(3), 997-1002

\title{
Chemometrics -Assisted Simultaneous Determination of Atenolol and Furosemide in Synthetic Binary Mixtures and Combined Tablet Preparations
}

\author{
AMIR H.M. SARRAFI ${ }^{*}$, ELAHE KONOZ and ALIREZA FEYZBAKHSH \\ *Department of Chemistry, Faculty of Science, \\ Islamic Azad University-Central Tehran Branch, \\ Simaye Iran St., San'at Square, Tehran, Iran. \\ amirsarrafi45@yahoo.com
}

Received 16 November 2009; Accepted 10 January 2010

\begin{abstract}
In this work a numerical method, based on the use of spectrophotometric data coupled to PLS multivariate calibration, is reported for the simultaneous determination of furosemide (FUR) and atenolol (ATE) in synthetic samples and combined commercial tablets. The correlation coefficients $\left(\mathrm{R}^{2}\right)$ and recovery range for ATE and FUR in synthetic mixtures were $0.9938,0.9949$ and $94.72-103.72 \%, 95.60-104.34 \%$ respectively. The results of optimized method in combined tablet preparations compared with British Pharmacopeia 2007 standard methods that have a good agreement. The proposed method are simple, fast, inexpensive and do not need to any separation or preparation methods.
\end{abstract}

Keywords: Atenolol, Furasemide, Spectrophotometric, Multivariate calibration.

\section{Introduction}

Atenolol (RS)-2-\{4-[2-hydroxy-3-(propan-2-ylamino)propoxy]phenyl $\}$ acetamide (ATE) is a selective $\beta_{1}$ receptor antagonist, a drug belonging to the group of beta blockers (sometimes written $\beta$-blockers), a class of drugs used primarily in cardiovascular diseases. Introduced in 1976, atenolol was developed as a replacement for propranolol in the treatment of hypertension. Furasemide 4-chloro-2-(furan-2-ylmethylamino)- 5-sulfamoylbenzoic acid (FUR) is a loop diuretic used in the treatment of congestive heart failure and edema. Along with some other diuretics, furosemide is also included on the World Anti-Doping Agency's banned drug list due to its alleged use as a masking agent for other drugs ${ }^{1}$. 
The determination of these drugs is a frequent analytical problem in quality control of the pharmaceutical industries. The two drugs studied in this work show a strong overlap between their absorption spectra. Hence, their simultaneous determination using conventional spectrophotometric techniques would be hard. Mainly, HPLC is used to resolve a complex mixture of these drugs ${ }^{2}$.

Partial Least Squares regression (PLS) was originally developed by Wold ${ }^{3}$ and the use of PLS method for chemical analysis was pioneered by Wold et $a l^{4-5}$. A detailed description on the mathematical principles of the PLS algorithms have been reported by Martens et al ${ }^{6}$.

In recent years multivariate calibration methods have been used to resolve mixtures of two or more compounds with similar spectra characteristics. In most cases, multivariate methods for evaluation of spectroscopic data have more advantages as simplicity and cheapness. These methods have been applied for determination of drugs ${ }^{7-11}$, because HPLC methods and conventional spectroscopic methods were slow, expensive and complex.

The aim of this paper is to investigate the ability of PLS method for quantifying binary mixtures of atenolol and furasemide without prior separation and to apply the optimized method in pharmaceutical preparations.

\section{Experimental}

Commercial samples were bought from pharmacies. Analytical grade ATE and FUR were obtained from Jalinous and Mehr-Daru pharmaceutical companies (Tehran, Iran). All other chemical and solvents were of analytical reagent grade.

\section{Apparatus and software}

A V-530 Jasco scanning spectrophotometer connected to PC fitted data software was used for all the measurements and treatment of data. The Matlab 7.1 and Unscrambler 9.1 softwares were used for the statistical treatment of the data and application of various multivariate methods.

\section{Procedure}

The $500 \mathrm{mg} / \mathrm{L}$ ATE and FUR stock solutions were prepared by dissolving accurately weighed amounts of finely powdered pure ATE and FUR in deionized water and $1.6 \times 10^{-3} \mathrm{M}$ $\mathrm{NaOH}$ respectively. The calibration and test mixtures prepared by mixing these two solutions with different ratios.

For $50 \mathrm{mg}$ ATE and $40 \mathrm{mg}$ FUR tablets, 20 of each tablets mixed and weighed. After grinding, mixing and homogenizing of an accurately weighed 20 tablets from each pharmaceutical products, 1/20 of sample was used for analysis. Each weighed sample was mixed with $40 \mathrm{~mL}$ deionized water and the mixture was subjected to ultrasonication for 15 minutes. After cooling and further dilution to $100 \mathrm{~mL}$ with deionized water, a $10 \mathrm{~mL}$ portion of the sample was centrifuged at $3000 \mathrm{rev} / \mathrm{min} .0 .25 \mathrm{~mL}$ portion of the supernatant was then diluted to $25 \mathrm{~mL}$ with deionized water.

For $100 \mathrm{mg}$ ATE and $40 \mathrm{mg}$ FUR tablets, 20 of each tablets mixed and weighed. After grinding, mixing and homogenizing of an accurately weighed 20 tablets from each pharmaceutical products, 1/40 of sample was used for analysis. Each weighed sample was mixed with $40 \mathrm{~mL}$ deionized water and the mixture was subjected to ultrasonication for 15 minutes. After cooling and further dilution to $100 \mathrm{~mL}$ with deionized water, a $10 \mathrm{~mL}$ portion of the sample was centrifuged at $3000 \mathrm{rev} / \mathrm{min} .0 .20 \mathrm{~mL}$ portion of the supernatant was then diluted to $25 \mathrm{~mL}$ with deionized water. The absorption spectra between 200 and $400 \mathrm{~nm}$ against blank were recorded for all solutions. Commercial tablets were also analyzed, using British Pharmacopoeia 2007 (BP2007) method ${ }^{12}$. 
The stability of ATE, FUR and commercial sample solutions were checked for $4 \mathrm{~h}$ and the UV-Vis absorption spectra of all sample solutions were found to be stable for this period of time. It is also to be noted that the simultaneous determination of the aforementioned drugs with the proposed method can be carried out in less than $1 \mathrm{~h}$.

\section{Results and Discussion}

\section{$U V$-Vis spectra of ATE and FUR}

In figure 1, the absorption spectra of ATE and FUR solutions in methanol recorded between 200 and $400 \mathrm{~nm}$ are shown. The two drugs studied show a strong overlap in their absorption spectra and the conventional spectrophotometric methods can not be applied for resolving this mixture.

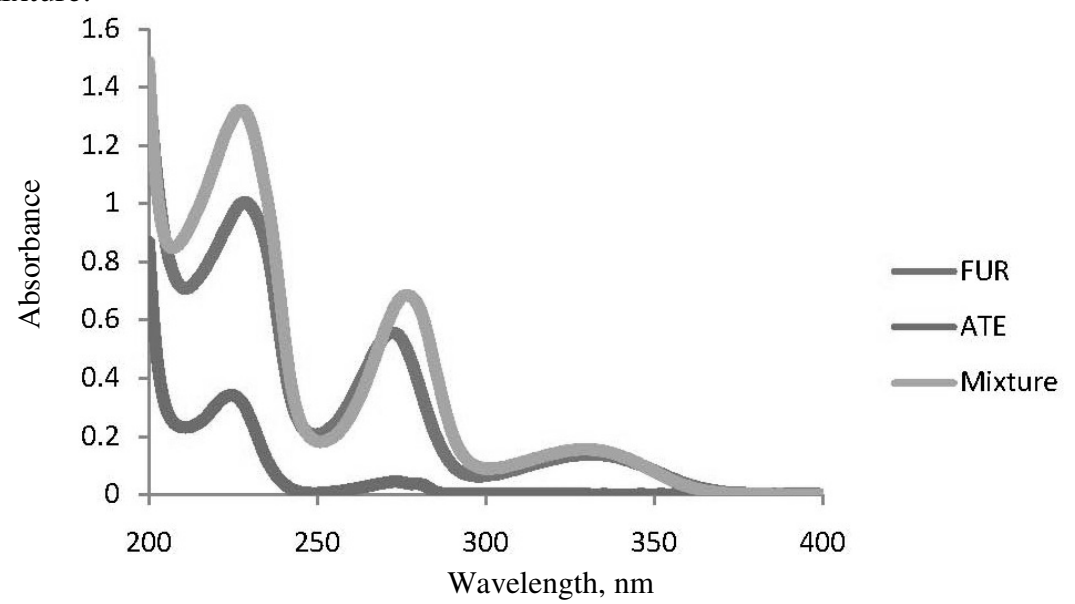

Figure 1. The UV-Vis absorption spectra ATE, FUR and mixture of tablets solutions.

\section{Experimental design of sample sets}

Calibration and test sets for two component systems were designed according to factorial principle. Solutions containing drug concentrations in the range $0.0-10.0 \mathrm{mg} / \mathrm{L}$ for ATE and FUR were produced by dilution of the stock solutions. A five-level factorial design was used to produce a full set of 25 samples. A three-level set was derived to produce a calibration set of nine samples, with the remaining 16 samples used for an independent test set ${ }^{13}$. The compositions of the used calibration and test sets are summarized in Table 1 and Table 2, respectively.

Table 1. Calibration set composition.

\begin{tabular}{ccc}
\hline Standard & ATE, mg/L & FUR, mg/L \\
\hline $\mathrm{C}_{1}$ & 0 & 0 \\
$\mathrm{C}_{2}$ & 0 & 5 \\
$\mathrm{C}_{3}$ & 0 & 10 \\
$\mathrm{C}_{4}$ & 5 & 0 \\
$\mathrm{C}_{5}$ & 5 & 5 \\
$\mathrm{C}_{6}$ & 5 & 10 \\
$\mathrm{C}_{7}$ & 10 & 0 \\
$\mathrm{C}_{8}$ & 10 & 5 \\
$\mathrm{C}_{9}$ & 10 & 10 \\
\hline
\end{tabular}


Table 2. Test set composition.

\begin{tabular}{ccc}
\hline Sample & ATE, mg/L & FUR, mg/L \\
\hline $\mathrm{T}_{1}$ & 0 & 2.5 \\
$\mathrm{~T}_{2}$ & 0 & 7.5 \\
$\mathrm{~T}_{3}$ & 2.5 & 0 \\
$\mathrm{~T}_{4}$ & 2.5 & 2.5 \\
$\mathrm{~T}_{5}$ & 2.5 & 5 \\
$\mathrm{~T}_{6}$ & 2.5 & 7.5 \\
$\mathrm{~T}_{7}$ & 2.5 & 10 \\
$\mathrm{~T}_{8}$ & 5 & 2.5 \\
$\mathrm{~T}_{9}$ & 5 & 7.5 \\
$\mathrm{~T}_{10}$ & 7.5 & 0 \\
$\mathrm{~T}_{11}$ & 7.5 & 2.5 \\
$\mathrm{~T}_{12}$ & 7.5 & 5 \\
$\mathrm{~T}_{13}$ & 7.5 & 7.5 \\
$\mathrm{~T}_{14}$ & 7.5 & 10 \\
$\mathrm{~T}_{15}$ & 10 & 2.5 \\
$\mathrm{~T}_{16}$ & 10 & 7.5 \\
\hline
\end{tabular}

Selection of the optimum number of factors and the spectral region

To select the correct number of factors in the PLS algorithm, a cross-validation method, leaving out one sample at a time, was employed. The predicted concentrations $\bar{X}_{i j}(k)$ of the compounds in each $l$ sample, obtained with $k$ factors, were compared with the already known concentrations $X i j$ and the root mean squared error of cross validation (RMSECV) was calculated for each of the $k$ factor levels as follows:

$$
R M S W C V(V)=\sqrt{\frac{\sum_{i=1}^{n} \sum_{j=1}^{m}\left(\bar{X}_{i j}(k)-X_{i j}\right)^{2}}{1}}
$$

The optional value for $k$ is the level that yields the smallest RMSECV $(\mathrm{k})$ value. To check the selected factor number, the external validation method was also used by simply computing the root mean squared error of predication (RMSEP) for $L$ objects in test set for each of the $k$ factor levels as follows:

$$
\operatorname{RMSEP}(k)=\sqrt{\frac{\sum_{i=1}^{n} \sum_{j=1}^{m}\left(\bar{Z}_{i j}(k)-Z_{i j}\right)^{2}}{L}}
$$

where $Z_{i j}$ is the known concentration in the test set and $\bar{Z}_{i j}(k)$ is predicted for concentrations with $k$ factors ${ }^{7}$. This showed that the external test set validation and the internal cross-validation indicated about the same number of factors.

To select the spectral region, all of the above steps used repeatedly and the spectral region that lead to the lowest values of RMSEP was selected ${ }^{7}$. The optimal number of factors, RMSEP and RMSECV values and optimum spectral regions obtained by PLS- 1 and PLS-2 algorithms are summarized in Table 3.

The proposed PLS-1 and PLS-2 calibration models were evaluated by prediction of drug concentrations in their own designed calibration set. Recoveries values were between 96.26 and $105.28 \%$ for ATE and between 95.52 and $103.57 \%$ for FUR. 
Table 3. Optimal number of factors, RMSEP and RMSECV values.

\begin{tabular}{cccccc}
\hline & & Factor No. & RMSEP & Factor No. & RMSECV \\
\hline PLS-1 & ATE & 2 & 0.2348 & 2 & 0.2406 \\
$212-243 \mathrm{~nm}$ & FUR & 2 & 0.2712 & 2 & 0.2971 \\
PLS-2 & ATE & 2 & 0.2299 & 2 & 0.2389 \\
$212-243 \mathrm{~nm}$ & FUR & 2 & 0.2355 & 2 & 0.2230 \\
\hline
\end{tabular}

Statistical parameters for the optimized models

Using the internal validation in their own designed calibration set, the following statistical parameters have been obtained:

a) The values of root mean squared error of calibration (RMSEC), which is an indication of the average error in the analysis for each component.

b) The square of correlation coefficients $\left(\mathrm{R}^{2}\right)$, which is an indication of the quality of the straight line that fits the data.

In Table 4, the results obtained for these parameters by PLS-1 and PLS-2 are shown. We can see that the $\mathrm{R}^{2}$ values are in all cases very near to 1 which is an indication of similarity between predicted and known values. On the other hand, in general terms, the statistical parameters obtained by PLS-1 and PLS-2 are good.

Table 4. Statistical parameters of the model optimized

\begin{tabular}{ccccc}
\hline & \multicolumn{2}{c}{ PLS-1 } & \multicolumn{2}{c}{ PLS-2 } \\
\cline { 2 - 5 } & RMSEC & $\mathrm{R}^{2}$ & RMSEC & $\mathrm{R}^{2}$ \\
\hline ATE & 0.2909 & 0.9921 & 0.2948 & 0.9919 \\
FUR & 0.2868 & 0.9970 & 0.1051 & 0.9993 \\
\hline
\end{tabular}

External validation of PLS-1 and PLS-2 calibration models

16 Synthetic mixtures in test set were predicted by applying PLS- 1 and PLS- 2 methods. The square of correlation coefficients $\left(\mathrm{R}^{2}\right)$ and recovery range for these synthetic solutions are summarized in Table 5. Satisfactory values are obtained in most of mixtures analyzed by the methods. Limits of detection (LOD's) were calculated as three times of standard error of estimation (SEE) values ${ }^{7}$. LOD's of $0.933 \mathrm{mg} / \mathrm{L}$ for ATE, $0.919 \mathrm{mg} / \mathrm{L}$ for FUR, $0.945 \mathrm{mg} / \mathrm{L}$ for ATE, and $0.929 \mathrm{mg} / \mathrm{L}$ for FUR were obtained in PLS-1 and PLS-2 models, respectively.

Table 5. Recovery range and $\mathrm{R}^{2}$ for synthetic mixtures in test set.

\begin{tabular}{ccccc}
\hline & \multicolumn{2}{c}{ ATE } & \multicolumn{2}{c}{ FUR } \\
\cline { 2 - 5 } \%Rec. range & $\mathrm{R}^{2}$ & \%Rec. range & $\mathrm{R}^{2}$ \\
\hline PLS-1 & $94.36-103.40$ & 0.9937 & $95.53-103.76$ & 0.9921 \\
PLS-2 & $94.72-103.72$ & 0.9938 & $95.60-104.34$ & 0.9949 \\
\hline
\end{tabular}

\section{Analysis of commercial samples}

Commercial mixture products were analyzed using two methods: the proposed spectrophotometric method and BP2007 method ${ }^{12}$. Results are summarized in Table 6. As can be seen, satisfactory results were obtained in all cases by the proposed methods.

Table 6. Analysis of commercial tablets mixtures.

\begin{tabular}{ccccc}
\hline & \multicolumn{2}{c}{ Commercial tablet mixtures } & \multicolumn{2}{c}{ Commercial tablet mixtures } \\
\cline { 2 - 5 } & ATE \pm RSD & FUR \pm RSD & ATE $\pm \%$ RSD & FUR $\pm \%$ RSD \\
\hline Declared contents* & 50 & 40 & 100 & 40 \\
PLS-1 Results & $50.11 \pm 1.01$ & $36.15 \pm 1.21$ & $102.58 \pm 1.01$ & $36.66 \pm 1.40$ \\
PLS-2 Results & $50.17 \pm 1.10$ & $36.55 \pm 1.00$ & $102.47 \pm 1.20$ & $36.38 \pm 1.10$ \\
BP2007 Results & $49.00 \pm 1.30$ & $36.00 \pm 1.40$ & $98.00 \pm 1.30$ & $36.00 \pm 1.40$ \\
\hline \multicolumn{5}{c}{ *Results presented as mg per tablet. }
\end{tabular}


Statistical parameters obtained by replicate analysis, for the commercial tablets with proposed PLS-1, PLS-2 and BP2007 standard methods are also given in Table 6. These results show that the precision of the proposed methods and BP2007 method are same.

\section{Conclusion}

A comparative study of the use of PLS- 1 and PLS-2 for the resolution and simultaneous determination of ATE and FUR in a binary mixture has been accomplished, showing that this method provide a clear example of the high resolving power of these techniques. In several terms, similar results were obtained for these two drugs in both synthetic and commercial mixture applications by PLS-1 and PLS-2.

The results obtained confirm the suitability of the proposed method for accurate and precise analysis of Atenolol and Furosemide in pharmaceutical preparations. These methods were applied directly to the commercial mixture preparations without previous treatment. Also no expensive (dissolution and injection) laboratory technique is needed. In addition the proposed methods are suitable for application without interference of the excipients.

\section{References}

1. www.fda.gov

2. Bansal T, Singh M, Mishra G, Talegaonkar S, Khar R K, Jaggi M and Mukherjee R, $J$ Chromatogr B, 2007, 859(2), 261-266.

3. Wold H, in Research Papers in Statistics, (David F, Eds), Wiley, New York, 1966.

4. Wold H, in Systems under Indirect Observations, (H. Joreskog and H. Wold, Eds), North-Holland, Amesterdam, 1982.

5. Wold S, Martens $\mathrm{H}$ and Wold $\mathrm{H}$, in The Multivariate Calibration Problem in Chemistry solved by PLS, Matrix Pencils (Lecture Notes in Mathematics), (A. Ruhe and Kagstrom B, Eds), Springer, Heidelberg, 1992.

6. Martens H and Naes T, Multivariate Calibration, Wiley, Chichester, 1992.

7. Kargosha K and Sarrafi A H M, J Pharm Biomed Anal., 2001, 26, 273-279.

8. Aksu O, Bozdogan A and Kunt G, Anal Lett., 2006, 39,751.

9. Hadad G M, El-Gindy A and Mahmoud W M M, Spectrochim Acta Part A, 2008, 70, 655-663.

10. Khoshayand M R, Abdollahi H, Shariatpanahi M, Saadatfard A and Mohammadi A, Spectrochim Acta Part A., 2008,70,491-499,

11. Sarrafi A H M, Khodakarami Z and Karkeabadi M, E Journal of Chemistry, 2009, 6(S1), S111-S116.

12. British Pharmacopeia 2007, CD-ROM.

13. Bayne C K and Rubin I B, Experimental Design, VCH, New York, 1986. 


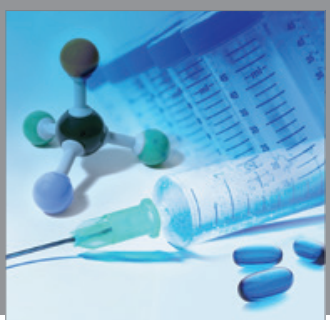

International Journal of

Medicinal Chemistry

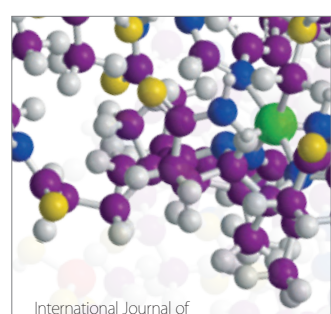

Carbohydrate Chemistry

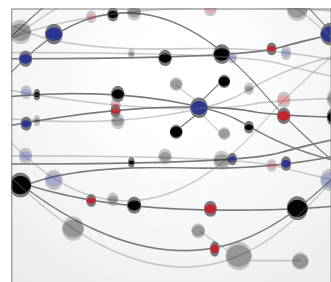

The Scientific World Journal
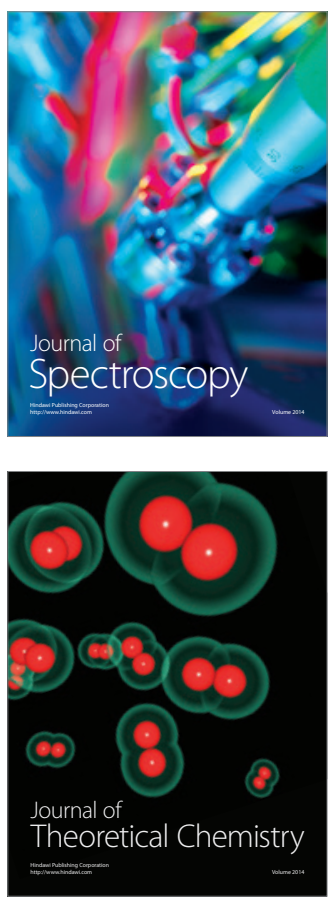
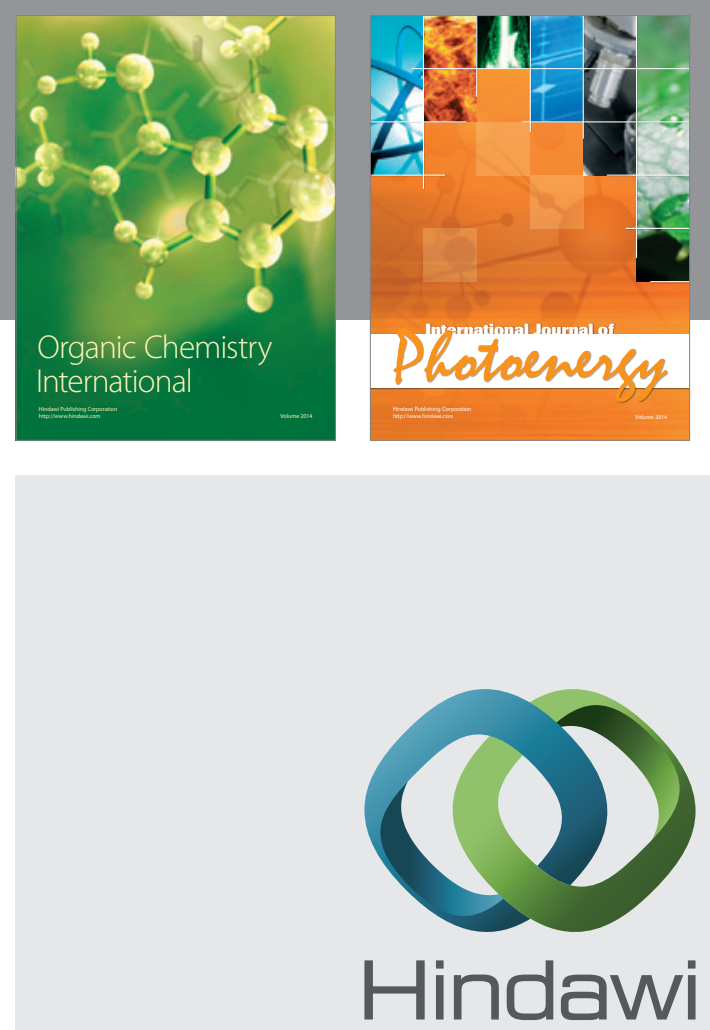

Submit your manuscripts at

http://www.hindawi.com
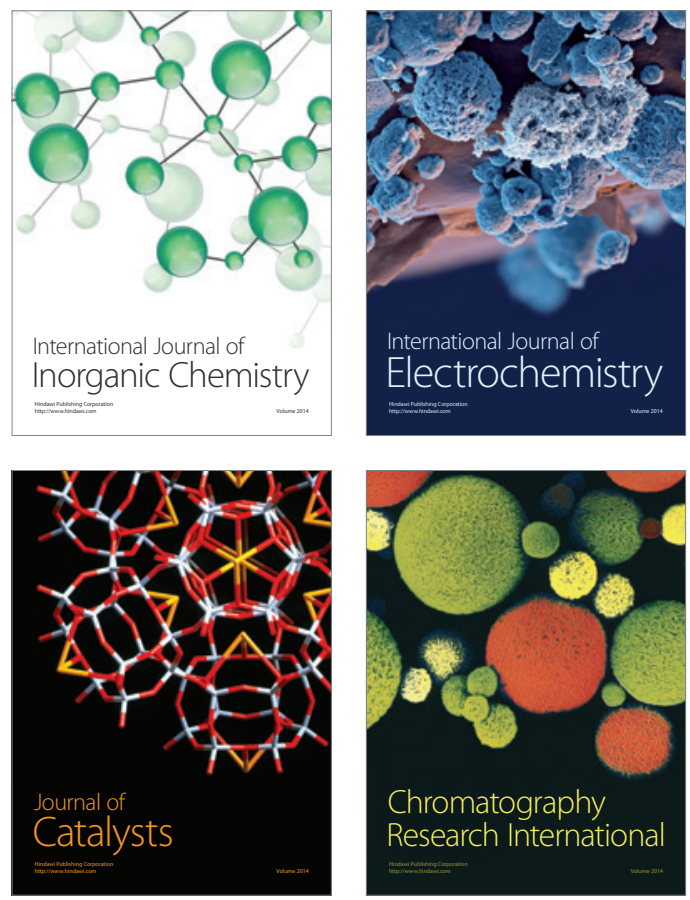
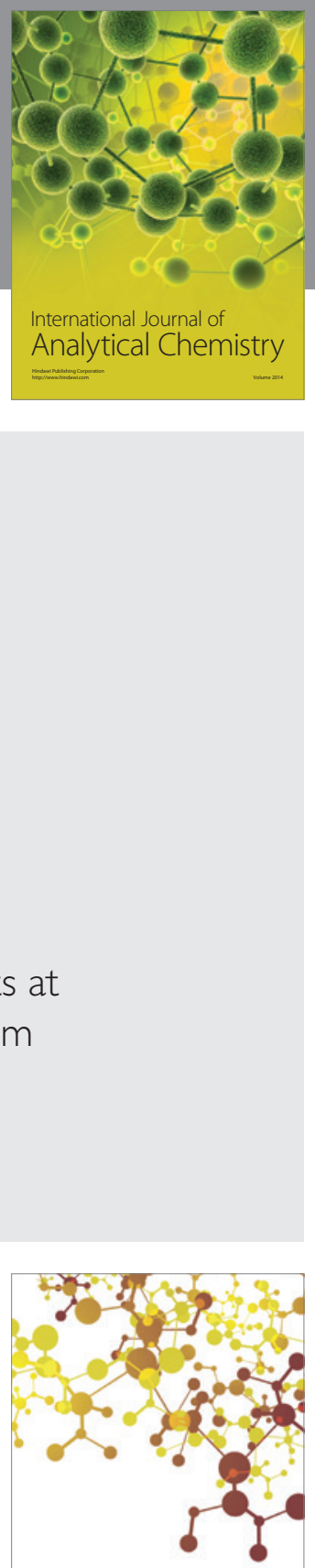

Journal of

Applied Chemistry
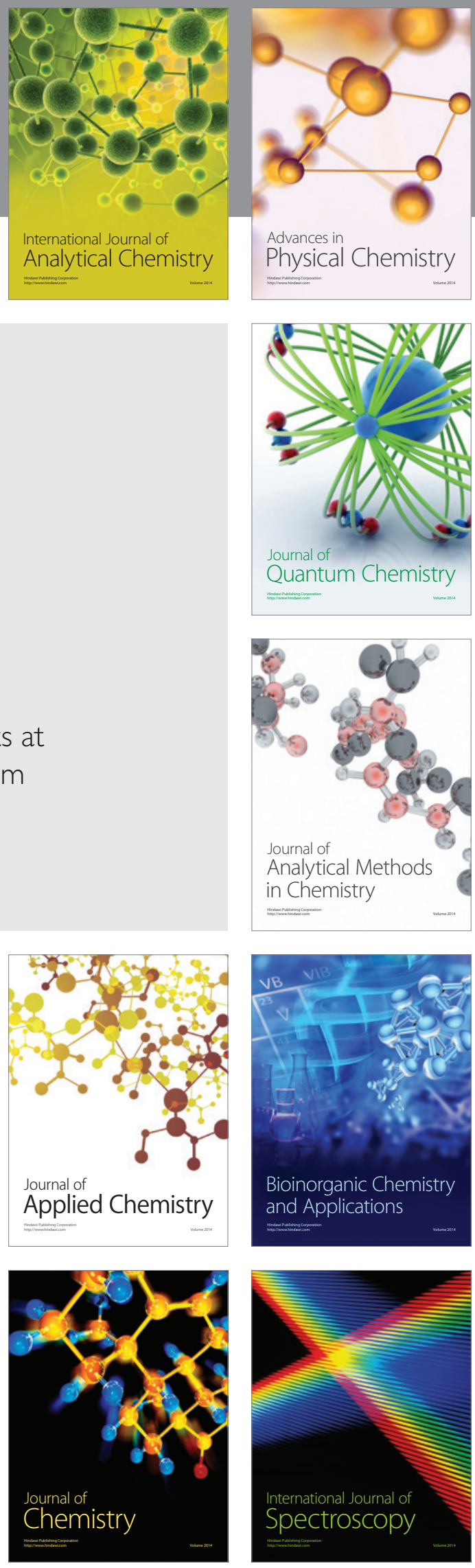